\title{
La collaboration dans la reconnaissance de l'autre
}

\section{Michel Matter}

Dr méd., vice-président de la FMH, Responsable du département Prestations et développement professionnel

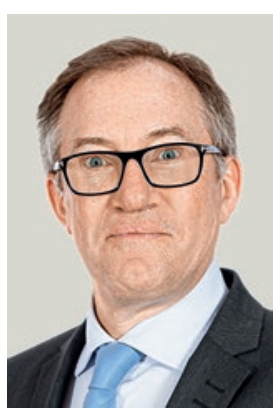

En avant-propos des «Perspectives à long terme des finances publiques en Suisse, 2021», le conseiller fédéral et chef du Département fédéral des finances, M. Ueli Maurer, souligne la pression démographique qui arrivera dans les prochaines années et avec elle les efforts financiers qu'il faudra effectuer dans les secteurs de la santé et de la prévoyance vieillesse. Le défi est de taille et les réformes sont indispensables. Au-delà du changement climatique, le vieillissement de la population doit devenir l'une des priorités en termes politiques et financiers.

La place des soignants va croître en importance. C'est là où notre système de santé doit être défendu et non attaqué, comme c'est le cas avec la volonté fédérale d'imposer un budget global. La prise en charge de seniors de plus en plus actifs, exigeants en termes de qualité de vie et de soins aigus ainsi que les accompagnements de fin de vie qui arrivent de plus en plus tardivement vont imposer des changements de paradigmes et de culture médicale. Une véritable interprofessionnalité et avec elle une coordination des soins sensiblement améliorée deviennent incontournables.

\section{Une véritable interprofessionnalité et avec} elle une coordination des soins sensiblement améliorée deviennent incontournables.

Les professions paramédicales se sont développées, spécialisées et ont gagné en compétence, en autonomie et en responsabilité au cours des dernières années. Cela est réjouissant et indispensable pour permettre de faire face à l'évolution démographique. La communication entre l'ensemble des acteurs de la santé doit être améliorée. L'arrivée, attendue comme Godot, d'un véritable dossier électronique partagé du patient qui contienne les informations médicales et paramédicales pertinentes et utiles, capable d'intégrer l'ensemble des systèmes électroniques actuellement utilisés dans les centres et cabinets médicaux est impérative. Mieux communiquer, mieux intégrer les différents avis et évaluations, mieux coordonner. Ce qui paraît l'évidence peine à avancer.

L'interprofessionnalité doit être enseignée tôt dans la formation par des troncs communs centrés sur la prise en charge commune du patient, mais elle ne pourra devenir réalité qu'en s'imposant au sein des pratiques

\section{L'interprofessionnalité doit être enseignée tôt} dans la formation par des troncs communs centrés sur la prise en charge commune du patient.

tant publiques que privées, des grandes et des petites structures de soins. C'est ensemble et dans le respect des compétences de l'autre que nous réussirons collectivement cette révolution, car c'en est bien une. Cette recherche de l'efficience et de l'efficacité doit s'inscrire dans notre propre agenda. C'est notamment une priorité stratégique du département Prestations et développement professionnel de la FMH, qui est très engagé dans le domaine.

Bien évidemment cela ne concerne pas l'ensemble des patientes et des patients, mais un grand nombre d'entre-eux. Là où certains vont évoquer le risque d'une explosion des coûts de la santé, il peut être aisément répondu que le vieillissement de la population, prévisible et planifiable, doit entraîner une réaction politique et financière, mais également de nouvelles pistes pour améliorer la prise en charge des patients et pouvoir réaliser des économies d'échelle. Chacune et chacun a ses propres compétences et le plus apte, selon son domaine de spécialisation, prend en charge le patient et sa situation clinique avec le concours actif de l'ensemble des autres professionnels de la santé, le but souhaité étant clairement établi.

Ensemble et avec respect. 